\title{
Pelger Huet Anomaly Measurement
}

National Cancer Institute

\section{Source}

National Cancer Institute. Pelger Huet Anomaly Measurement. NCI Thesaurus. Code C74617.

The determination of the amount of Pelger Huet anomaly present in a sample. 